\title{
Role of the thioredoxin interacting protein in diabetic nephropathy and the mechanism of regulating NOD-like receptor protein 3 inflammatory corpuscle
}

\author{
CHUNMEI GU ${ }^{1}$, SHUMEI LIU ${ }^{2}$, HONGYUE WANG ${ }^{1}$ and HAICHUAN DOU ${ }^{1}$
}

Departments of ${ }^{1}$ Nephrology and ${ }^{2}$ Endocrinology, The First Hospital of Jilin University, Changchun, Jilin 130021, P.R. China

Received August 15, 2018; Accepted April 2, 2019

DOI: $10.3892 / \mathrm{ijmm} .2019 .4163$

\begin{abstract}
Inflammatory response serves an important role in diabetic nephropathy (DN); however, the mechanism of inflammatory response results in renal damage is not yet clear. The aim of the present study was to investigate the role of the thioredoxin interacting protein (TXNIP)/NOD-like receptor protein 3 (NLRP3) axis-mediated activation of NLRP3 inflammasome in DN. A diabetic rat model was induced by streptozotocin injection. Hematoxylin and eosin (H\&E) staining and streptavidin-peroxidase staining were then used to examine the kidney tissue morphology, and TXNIP and NLRP3 protein expression levels, respectively. Furthermore, RNA interference technology was applied to silence the TXNIP gene. TXNIP and NLRP3 inflammasome activation-associated proteins and mRNAs were detected by western blot analysis and RT-qPCR, respectively. Enzyme-linked immunosorbent assay was also used to examine interleukin (IL)-1 and IL-18 expression, while flow cytometry was performed to detect reactive oxygen species production. The data revealed that TXNIP and NLRP3 were overexpressed in kidney tissue of DN rats, and the level of antioxidant genes was downregulated. It was also observed that glucose promoted TXNIP expression and activation of NLRP3 inflammasome in a time-dependent and dose-dependent manner, therefore promoting inflammatory responses. Silencing of TXNIP gene resulted in the downregulation of NLRP3 inflammasome activation, and inhibited the expression levels of IL-1 and IL-18 in a high-glucose environment. Furthermore, low expression of TXNIP promoted the levels of antioxidant genes and reduced the ROS levels. Taken together, the high-glucose environment was able to upregulated the level of inflammatory factors by promoting the expression of TXNIP and the activation of NLRP3 inflammasome, consequently participating in DN.
\end{abstract}

Correspondence to: Dr Haichuan Dou, Department of Nephrology, The First Hospital of Jilin University, 3302 Jilin Road, Erdao, Changchun, Jilin 130021, P.R. China

E-mail: douhaichuan_dhc@163.com

Key words: diabetic nephropathy, NOD-like receptor protein 3, thioredoxin interacting protein, oxidative, inflammatory

\section{Introduction}

The incidence of diabetes is increasing annually (1). Diabetic nephropathy (DN) is a common and serious complication of diabetes, and one of the main causes of mortality in diabetic patients (2). To reduce the risk of DN, it is very important to study the pathogenesis of DN and implement appropriate drug control; however, the pathogenesis of DN currently remains unclear. Previous studies have suggested that inflammatory and oxidative stress reactions are involved in the development of DN (2-4). The NOD-like receptor protein 3 (NLRP3) inflammasome activates inflammatory factors, and NLRP3 inflammasome activation induced by reactive oxygen species (ROS) may be involved in the occurrence and development of kidney injury.

Elevated levels of ROS are common in diabetic patients, and these species are necessary for the activation of NLRP3 inflammasomes. Upon the activation of NLRP3 inflammasomes, NLRP3 activates caspase-1, and then interleukin (IL)-1 and IL-18 are cleaved and activated, leading to pro-inflammatory responses $(5,6)$. NLRP3, IL-1 and IL-18 are closely associated with various diseases, including kidney disease $(7,8)$. Although the mechanism of NLRP3 inflammasome activation has been one of the research hotspots in recent years, there are few studies on the activation of NLRP3 in DN.

Thioredoxin interacting protein (TXNIP) belongs to the thioredoxin system and is an important participant in regulating oxidative stress in the body (9). Thioredoxin (TRX) is the main molecule that resists oxidative stress in cells. TXNIP can bind to TRX, decreasing TRX activity and increasing oxidative stress (9). It has been reported that TXNIP is able to activate NLRP3 inflammasome by binding to NLRP3 (10). However, the association between TXNIP and NLRP3 inflammasome in DN is not well understood.

The current study mainly investigated the expression characteristics of NLRP3 in DN, and examined the effect of TXNIP on oxidative stress and NLRP3 expression. Furthermore, the role of TXNIP gene in the occurrence and development of DN was assessed.

\section{Materials and methods}

Animals and establishment of a diabetes mouse model. A total of 25 5-6 week old male Sprague-Dawley rats were purchased 
from Shanghai Laboratory Animal Center (Shanghai, China). Rats were fed a normal diet with water ad libitum prior to treatment administration and kept under a $12 \mathrm{~h}$ light/dark cycle in a controlled environment (temperature, $22 \pm 2^{\circ} \mathrm{C}$; humidity 60-80\%). All protocols, including diabetes induction and animal sacrifice, were approved by the Institutional Animal Care and Use Committee of the First Hospital of Jilin University (Changchun, China), and the China Council on Animal Care. The rats received adaptive feeding.

Rats in DN group $(n=15)$ were administered an intraperitoneal injection of streptozotocin $(55 \mathrm{mg} / \mathrm{kg})$, while rats in the control group $(n=10)$ received only citrate buffer. Biochemical kits (Xibao Biotechnology Co., Ltd., Shanghai, China) were used to measure the blood glucose levels at 24,48 and $72 \mathrm{~h}$ after streptozotocin injection. After $72 \mathrm{~h}$ of assessment, blood was collected from the tail vein of rats and fasting blood glucose was determined. Rats with fasting blood glucose levels of $>250 \mathrm{mg} / \mathrm{dl}$ were considered as diabetic. The levels of blood urea nitrogen (BUN) and serum creatinine ( $\mathrm{SCr})$ in rats were detected by enzyme-linked immunosorbent assay (ELISA), and the corresponding kits were purchased from Wuhan Huamei Biotech Co., Ltd. (Wuhan, China).

Hematoxylin and eosin $(H \& E)$ staining. $H \& E$ staining was performed to observe the kidneys in the control and DN groups. After $72 \mathrm{~h}$ of injection, the rats were sacrificed and kidney tissue samples were collected at the end of week 1 , 2 and 3 . Kidney tissues from rats were immediately fixed in formalin for $24 \mathrm{~h}$ at room temperature. Next, tissues were dehydrated with alcohol, embedded in paraffin, cut into $7-\mu \mathrm{m}$ uniform sections and placed on glass slides coated with 3-aminopropyltriethoxysilane. The sections were then deparaffinized using xylene, hydrated and stained with H\&E reagent (Sigma-Aldrich; Merck KGaA, Darmstadt, Germany). For staining, sections were incubated with hematoxylin at room temperature for $5 \mathrm{~min}$, washed and then incubated with eosin at room temperature for 1-3 min. Subsequent to washing with gradient ethanol, neutral gel was used for sealing. The mean glomerular volume was calculated by measuring the maximum diameter of the glomerulus in 10 random fields.

Streptavidin-peroxidase (SP) staining. SP staining was performed to detect the TXNIP and NLRP3 protein expression levels. Briefly, tissue specimens were cut into $4-\mu \mathrm{m}$ section and deparaffinized in xylene. Next, $0.01 \mathrm{~mol} / 1$ citrate buffer solution was used for antigen retrieval, and $50 \mu \mathrm{l}$ peroxidase blocking solution was added to block the endogenous peroxidase activity. The primary antibodies (anti-NLRP3, 1:500; ab214185; anti-TXNIP, 1:200; ab188865; Abcam, Cambridge, MA, USA) were added and incubated at $4^{\circ} \mathrm{C}$ for $12 \mathrm{~h}$. Subsequently, the horseradish peroxidase conjugated secondary antibody (goat anti-rabbit IgG; 1:200; ab205718; Abcam) was added and incubated at room temperature for $10 \mathrm{~min}$. DAB $(100 \mu \mathrm{l})$ was then added for $5 \mathrm{~min}$, with hematoxylin being used for counterstaining at room temperature for $2 \mathrm{~min}$, and the staining was observed under a microscope. The cell staining intensity in three random fields of view was calculated using a semi-quantitative method $(11,12)$.
Cell culture. Human proximal tubular epithelial HK-2 cells were purchased from the American Type Culture Collection (Manassas, VA, USA). The cells were cultured in Dulbecco's modified Eagle's medium containing $10 \%$ fetal bovine serum at $37^{\circ} \mathrm{C}$ in an incubator with $5 \% \mathrm{CO}_{2} . \mathrm{HK}-2$ cells were divided into the control group, high-glucose group 1 (HG1), HG2 and HG3, and then cultured with $5.5,15,30$ or $50 \mathrm{mmol} / 1$ glucose, respectively. HK-2 cells in each group were cultured with glucose for $24,48,72$ or $96 \mathrm{~h}$ at $37^{\circ} \mathrm{C}$. These cell groups were subjected to western blot analysis and ELISA. Based on the results of these analyses, the experimental conditions of HG3 group (50 mmol/1 glucose) were used in subsequent experiments, and this group was defined as HG. Reagents used in cell culture were purchased from Thermo Fisher Scientific, Inc. (Gibco; Waltham, MA, USA).

Small interfering RNA (siRNA) construction, transfection and grouping. siRNA targeting TXNIP (siTXNIP) was purchased from GenePharma Co., Ltd. (Shanghai, China). siRNA transfection of cells was performed using Lipofectamine ${ }^{\circledR} 2000$ reagent (Invitrogen; Thermo Fisher Scientific, Inc.) to obtain the siTXNIP group. A NC group transfected with NC-siRNA and an untransfected control group were also established. To assess the effect of TXNIP gene silencing on HK-2 cells under a high-glucose environment, cells were divided into five groups as follows: A control group, A HG group $(50 \mathrm{mmol} / \mathrm{l}$ glucose), a negative $+\mathrm{HG}$ group $(\mathrm{NC}+\mathrm{HG}$; cells transfected with NC-siRNA + $50 \mathrm{mmol} / \mathrm{l}$ glucose), an siTXNIP + HG group (cells transfected with siTXNIP + $50 \mathrm{mmol} / \mathrm{l}$ glucose) and an siTXNIP group (cells transfected with siTXNIP).

Western blot analysis. Protein expression was assessed by western blot analysis. Briefly, cells were lysed with liquid nitrogen and blocked with radioimmunoprecipitation assay reagent (Abmole Bioscience, Inc., Houston, TX, USA), followed by $1 \%$ cleavage in phenylmethane sulfonyl fluoride and phosphatase inhibitors (Abmole Bioscience, Inc.), and lysis for $30 \mathrm{~min}$ at $4^{\circ} \mathrm{C}$. The supernatant was collected by centrifugation at $12,000 \mathrm{x} \mathrm{g}$ at $4^{\circ} \mathrm{C}$ for $15 \mathrm{~min}$, and a standard curve was constructed using the bicinchoninic acid method to determine the protein concentration. A 10\% SDS-PAGE gel was then prepared and used for electrophoresis. Next, samples were transferred to a polyvinylidene difluoride membrane using a Trans-Blot Transfer Slot (Bio-Rad Laboratories, Inc., Hercules, CA, USA) and blocked with 5\% fat-free milk for $2 \mathrm{~h}$ at room temperature. Primary antibodies were added, shaken at room temperature for $2 \mathrm{~h}$ and then incubated at $4^{\circ} \mathrm{C}$ for $12 \mathrm{~h}$. The primary antibodies used were as follows: Anti-NLRP3 (ab214185; dilution, 1:800), anti-TXNIP (ab188865; dilution, 1:600), anti-caspase-1 (ab138483; dilution, 1:800), anti-IL-1 (ab150777; dilution, 1:600), anti-IL-18 (ab52914; dilution, 1:600), anti-catalase (ab16731; dilution, 1:800) and anti-manganese superoxide dismutase (MnSOD; ab13533; dilution, 1:800), all purchased from Abcam; and anti-cleaved-caspase-1 (Orbyk310040; dilution, 1:700), supplied by Xiamen Research Biotechnology Co., Ltd. (Xiamen, China). Subsequently, secondary antibodies were added and incubated at room temperature for $1.5 \mathrm{~h}$. The secondary antibodies used were as follows: Goat anti-rat IgG (ab150160; dilution, 1:10,000), donkey anti-rat IgG (ab175475; 
Table I. Sequences of primers used in polymerase chain reaction.

\begin{tabular}{llc}
\hline Gene & \multicolumn{1}{c}{ Primer sequence (5'-3') } & Product size (bp) \\
\hline Catalase & Forward: GGTGCGGACATTCTACACAAAG & \\
& Reverse: TGTTCTCACACAGGCGTTTCC & 252 \\
MnSOD & Forward: ACCTGAGCCCTAATGGTGGTGGAGA & \\
& Reverse: ATTGAAACCGAGCCAGCCCCACCCA & 149 \\
GAPDH & Forward: CCATCTTCCAGGAGCGAGAT & \\
& Reverse: TGCTGATGATCTTGAGGCTG & 222 \\
\hline
\end{tabular}

MnSOD, manganese superoxide dismutase.

dilution, 1:8,000), rabbit anti-mouse IgG (ab99697; dilution, 1:9,000) and rabbit anti-human IgG (ab6759; dilution, 1:10,000), all purchased from Abcam; and mouse anti-rabbit IgG (31213; dilution, 1:7,000), obtained from Thermo Fisher Scientific, Inc. (Invitrogen). Chemiluminescence detection was subsequently conducted using an ECL reagent (Huiying Medical Technology Co., Ltd., Shanghai, China). Optical density was quantified using ImageJ software version 1.46 (National Institutes of Health, Bethesda, MD, USA).

ELISA. The malondialdehyde (MDA), superoxide dismutase (SOD), IL-1 and IL-18 concentrations of rat serum were assessed by ELISA assay using kits purchased from Nanjing Jiancheng Bioengineering Institute (Nanjing, China). Briefly, subsequent to incubation with blocking solution at $4^{\circ} \mathrm{C}$ for $2 \mathrm{~h}$, the primary antibody was added and incubated at $4^{\circ} \mathrm{C}$ overnight. The secondary antibody was then added and incubated for $1 \mathrm{~h}$ at room temperature. Horseradish peroxidase (100 $\mu \mathrm{l})$ was added and incubated at room temperature for $30 \mathrm{~min}$, followed by addition of 3,3',5,5'-tetramethylbenzidine for $10 \mathrm{~min}$. The absorbance value was measured at $450 \mathrm{~nm}$ by a microplate reader (ELX 800; BioTek Instruments, Inc., Winooski, VT, USA), and the concentration of each compound was calculated according to the standard curve.

Reverse transcription-quantitative polymerase chain reaction (RT-qPCR). RT-qPCR was performed to detect the mRNA levels. The cells were triturated and lysed using TRIzol reagent (Thermo Fisher Scientific, Inc.) at $0^{\circ} \mathrm{C}$ for $5 \mathrm{~min}$, and the RNAs were extracted by $\mathrm{CHCl}_{3}$ (Shanghai Aladdin Technology Co., Ltd., Shanghai, China). Next, the RNA concentration was measured by a UV spectrophotometer (NanoDrop One Microvolume UV-Vis spectrophotometer; Thermo Fisher Scientific, Inc.). RT assays were performed on RNA samples using an RT kit (Takara Bio, Inc.) to synthesize cDNA. The RT reaction conditions were set to $37^{\circ} \mathrm{C}$ for $15 \mathrm{~min}$, and reverse transcriptase inactivation was conducted at $85^{\circ} \mathrm{C}$ for $15 \mathrm{sec}$. Subsequently, qPCR experiments were performed with the SYBR Premix Ex Taq ${ }^{\mathrm{TM}}$ Real-Time PCR kit (Takara Bio, Inc.). qPCR was performed by activating the DNA polymerase at $95^{\circ} \mathrm{C}$ for $5 \mathrm{~min}$, followed by 40 cycles of two-step PCR (at $95^{\circ} \mathrm{C}$ for $10 \mathrm{sec}$ and $60^{\circ} \mathrm{C}$ for $30 \mathrm{sec}$ ) and a final extension at $75^{\circ} \mathrm{C}$ for $10 \mathrm{~min}$, held at $4^{\circ} \mathrm{C}$. DNase and RNase-free water were used as the templates of negative control experiments. All primers were obtained from Genewiz (Suzhou, China) and are listed in Table I. GAPDH was used as an internal control. The formula $2^{-\Delta \Delta \mathrm{Cq}}$ (13) was implemented to analyze and quantify the gene expression.

Flow cytometry. Flow cytometry was applied to detect ROS levels using ROS assay kits purchased from BD Biosciences (BD Pharmingen; San Jose, CA, USA). Briefly, cells were seeded in 6-well plates $\left(1 \times 10^{6}\right.$ cells/well), washed with PBS at $4^{\circ} \mathrm{C}$ and resuspended to a concentration of $4 \times 10^{5}$ cells $/ \mathrm{ml}$. Next, $10 \mu \mathrm{mol} / 1$ of the fluorescent probe 2',7'-dichlorodihydrofluorescein diacetate was added and incubated for $20 \mathrm{~min}$. A flow cytometer (BD Biosciences) was applied to analyze the ROS levels, at an excitation wavelength of $488 \mathrm{~nm}$ and an emission wavelength of $525 \mathrm{~nm}$.

Statistical analysis. All the experimental data are presented as the mean \pm standard deviation. Statistical analyses were conducted with SPSS software, version 20 (IBM, Corp., Armonk, NY, USA). One-way analysis of variance, followed by Turkey's multiple comparison, was applied to analyze differences among the experimental groups. $\mathrm{P}<0.05$ was considered to indicate a statistically significant difference.

\section{Results}

Changes on renal tissue and blood glucose in DN group. Alterations in rat kidney tissue at 1,2 and 3 weeks after streptozotocin injection were subsequently assessed using H\&E staining. In the control group, glomerular and tubular tissues were observed to be normal. However, in the DN group, evident necrosis of the kidneys and widening of the mesangial area were observed. Cystic edema also appeared in the kidney tubular epithelial cells (Fig. 1B). Furthermore, significantly increased glomerular volume (Fig. 1C) and vacuolar degeneration were also observed in DN rats. At 24, 48 and $72 \mathrm{~h}$ after streptozotocin administration, the blood glucose level of rats was significantly higher compared with that of the control group. Furthermore, the level of blood glucose in the DN group were $>250 \mathrm{mg} / \mathrm{dl}$ (Fig. 1A), indicating that the diabetic rat model was established successfully. As shown in Table II, BUN and SCr levels were significantly increased in the DN group compared with the control. These results demonstrated that kidney damage occurred in rats of the DN group. 
A

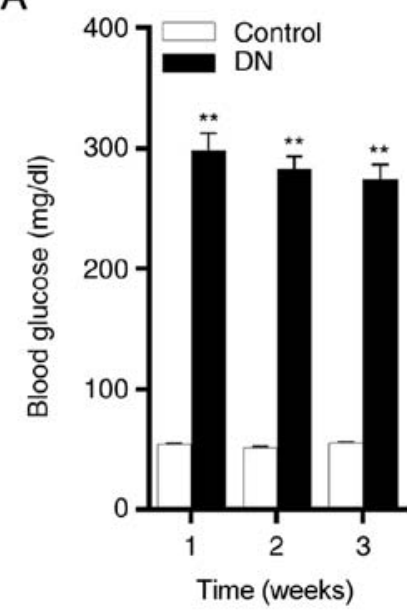

B

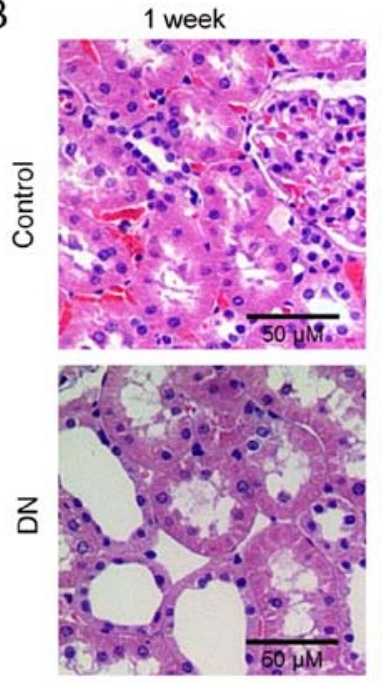

2 weeks
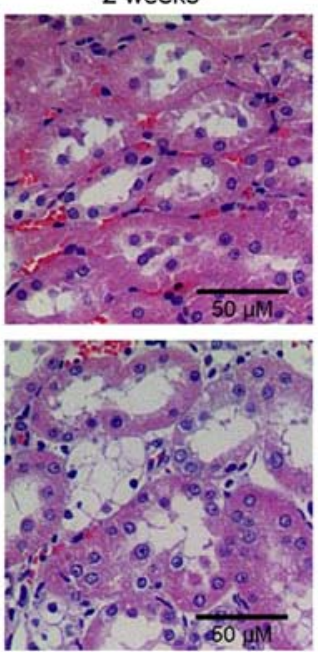

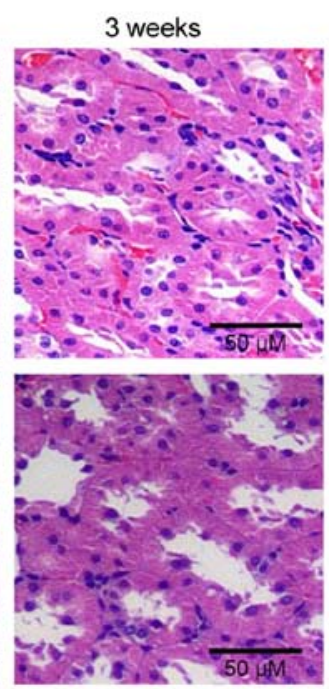

C

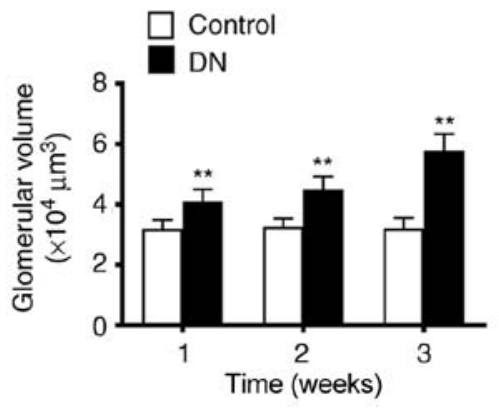

Figure 1. Changes in blood glucose and renal tissue morphology in rats following streptozotocin injection. (A) Blood glucose level changes at 1,2 and 3 weeks after the injection with streptozotocin. (B) Hematoxylin and eosin staining of the kidney tissues of the rats was observed under a microscope (magnification, $\mathrm{x} 100)$. (C) Glomerular volume was assessed at 1,2 and 3 weeks after streptozotocin injection. ${ }^{* *} \mathrm{P}<0.01$, vs. control group. DN, diabetic nephropathy.

Expression levels of TXNIP, NLRP3 and inflammatory factors in kidney tissue of DN rats. Western blot analysis was performed to detect the levels of TXNIP and NLRP3 inflammasome proteins, as well as of a number of inflammatory factors. The results demonstrated that the levels of TXNIP, NLRP3, cleaved-caspase-1, IL-1 and IL-18 in the DN group were significantly higher compared with those in the control group $(\mathrm{P}<0.05$; Fig. $2 \mathrm{~A}$ and $\mathrm{B})$. This indicated that the high-glucose level promoted TXNIP expression and NLRP3 inflammasome activation. Furthermore, SP staining results revealed that the expression levels of TXNIP and NLRP3 in the kidney tissue of DN rats markedly increased (Fig. 2C and D), indicating that the high glucose promoted TXNIP and NLRP3 protein expression levels.

Expression levels of antioxidant genes, MDA and SOD in $D N$ group. The protein and mRNA expression levels of catalase and MnSOD were detected by western blot analysis and RT-qPCR, respectively. The experimental results demonstrated that catalase and MnSOD protein and mRNA expression levels in the DN group were significantly lower in comparison with those in the control group $(\mathrm{P}<0.01$; Fig. 3A-C). ELISA results also revealed that the SOD expression level was significantly reduced in the DN group, while MDA level was markedly increased (Fig. 3D and E). These results indicated that the antioxidant capacity of kidneys in DN rats was reduced.
Effect of different glucose concentrations on HK-2 cells. Western blot analysis was performed to detect the expression levels of TXNIP, NLRP3, cleaved-caspase-1 and caspase-1 proteins in HK-2 cells in the control, HG1,HG2 and HG3 groups. The results demonstrated that as the concentration of glucose in the medium increased, the levels of TXNIP, NLRP3 and cleaved-caspase-1 proteins were gradually increased, while the level of caspase-1 protein decreased (Fig. 4A and B). The ELISA results also revealed that the levels of IL-1 and IL-18 secretion increased as the concentration of glucose was raised in the medium (Fig. 4C and D). This indicated that glucose promoted the expression of TXNIP and the activation of NLRP3 inflammasome, as well as enhanced the inflammatory response, in a dose-dependent manner.

Effects of the high-glucose on HK-2 cells at different time points. Western blot analysis was further performed to detect the expression levels of TXNIP, NLRP3, cleaved-caspase-1 and caspase- 1 proteins in HK-2 cells in the HG3 group at 0 , 24, 48, 72 and $96 \mathrm{~h}$. The results demonstrated that, as time progressed, the levels of TXNIP, NLRP3 and cleaved-caspase-1 protein were gradually increased, reaching a peak value at $\sim 72 \mathrm{~h}$ (Fig. 5A and B). By contrast, caspase-1 protein levels were not markedly altered. The ELISA results also demonstrated that the levels of IL-1 and IL-18 secretion significantly increased as time progressed (Fig. 5C and D). These findings indicated that glucose upregulated the expression of TXNIP in 
Table II. Changes in BUN and SCr in rat DN model.

\begin{tabular}{llrrr}
\hline Time & Parameter & Control & DN & P-value \\
\hline 1 week & BUN (mmol/l) & $7.83 \pm 0.73$ & $14.96 \pm 0.94$ & $<0.001$ \\
& SCr $(\mu \mathrm{mol} / \mathrm{l})$ & $37.44 \pm 1.15$ & $46.85 \pm 1.42$ & $<0.001$ \\
2 weeks & BUN $(\mathrm{mmol} / \mathrm{l})$ & $8.04 \pm 0.79$ & $18.56 \pm 1.67$ & $<0.001$ \\
& SCr $(\mu \mathrm{mol} / \mathrm{l})$ & $38.17 \pm 1.72$ & $55.79 \pm 1.78$ & $<0.001$ \\
3 weeks & BUN (mmol/l) & $8.21 \pm 0.81$ & $21.83 \pm 1.59$ & $<0.001$ \\
& SCr $(\mu \mathrm{mol} / \mathrm{l})$ & $39.08 \pm 2.73$ & $62.85 \pm 1.94$ & $<0.001$ \\
\hline
\end{tabular}

DN, diabetic nephropathy; BUN, blood urea nitrogen; SCr, serum creatinine.

A

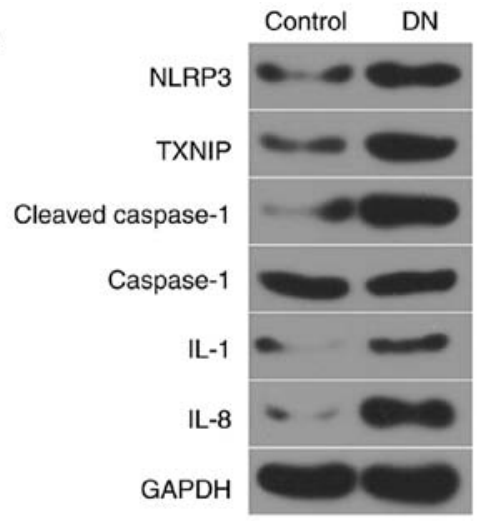

B

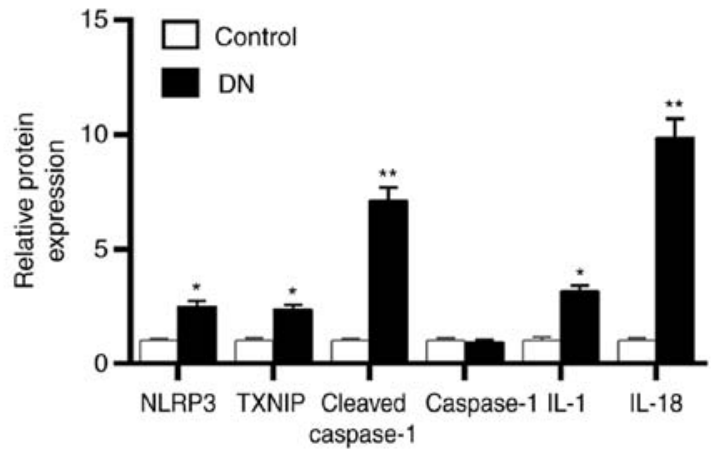

C

Control
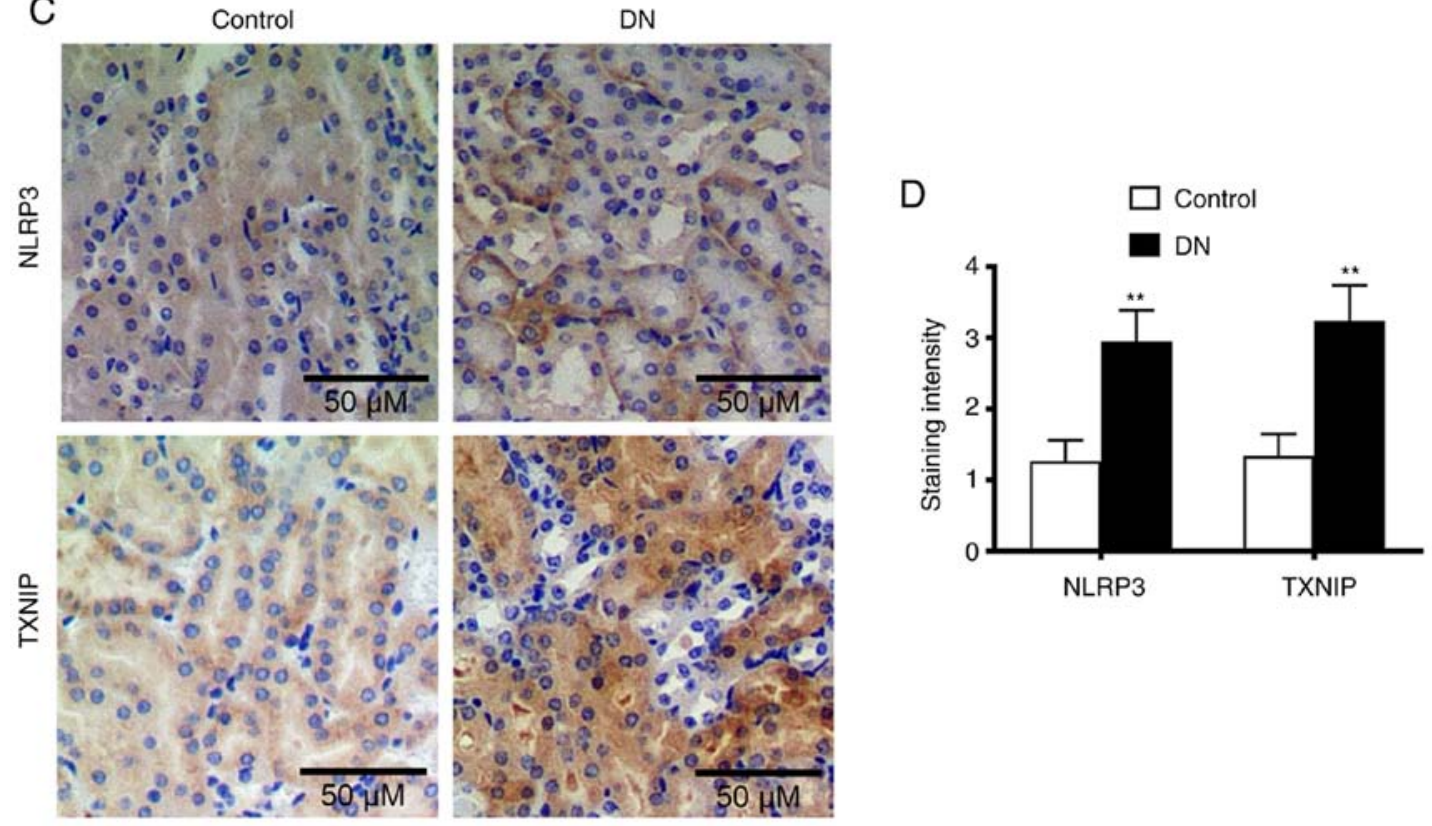

Figure 2. Expression levels of TXNIP and NLRP3 inflammasome-associated proteins in renal tissue of DN rats. (A) Western blot images and (B) quantified protein levels of TXNIP, NLRP3, cleaved-caspase-1, caspase-1, IL-1 and IL-18 in the control and DN groups. (C) Streptavidin-peroxidase staining was used to detect the TXNIP and NLRP3 proteins expression in kidneys tissues (magnification, $x 100$ ), and (D) the quantified staining intensity is shown. ${ }^{*} \mathrm{P}<0.05$ and ${ }^{* *} \mathrm{P}<0.01$, vs. control group. DN, diabetic nephropathy; TXNIP, thioredoxin interacting protein; NLRP3, NOD-like receptor protein 3; IL, interleukin.

a time-dependent manner, promoted the activation of NLRP3 inflammasome and enhanced the inflammatory response.

Effects of TXNIP gene silencing on HK-2 cells under a high-glucose environment. Western blot analysis was applied to detect the protein expression levels of TXNIP, NLRP3 inflammasome, and inflammation-associated factors in the control, $\mathrm{HG}, \mathrm{NC}+\mathrm{HG}$, siTXNIP and siTXNIP + HG groups. The results revealed that the TXNIP, NLRP3 and cleaved-caspase-1 protein levels were upregulated in the HG and $\mathrm{NC}+\mathrm{HG}$ groups compared with the control group $(\mathrm{P}<0.01)$. By contrast, the protein levels of TXNIP and cleaved-caspase-1 
A

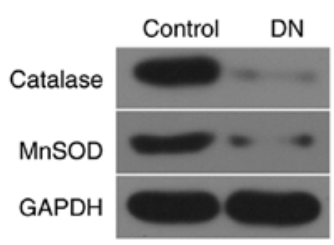

B

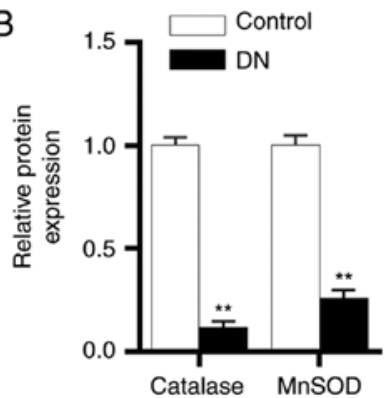

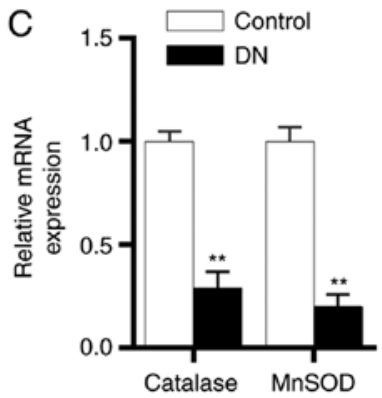
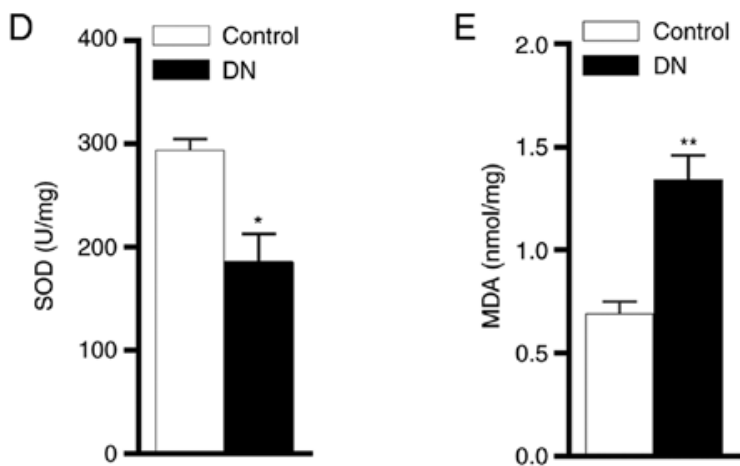

Figure 3. Antioxidant gene expression levels in DN rats. (A) Western blots and (B) quantified protein expression levels of catalase and MnSOD proteins in renal tissues in the control and DN groups were detected by performing western blot analysis. (C) mRNA expression levels of catalase and MnSOD in renal tissues in the two groups were detected by reverse transcription-quantitative polymerase chain reaction. (D) SOD and (E) MDA concentrations in the two groups, detected using ELISA. ${ }^{*} \mathrm{P}<0.05$ and ${ }^{* *} \mathrm{P}<0.01$ vs. control group. DN, diabetic nephropathy; MnSOD, manganese superoxide dismutase; MDA, malondialdehyde.
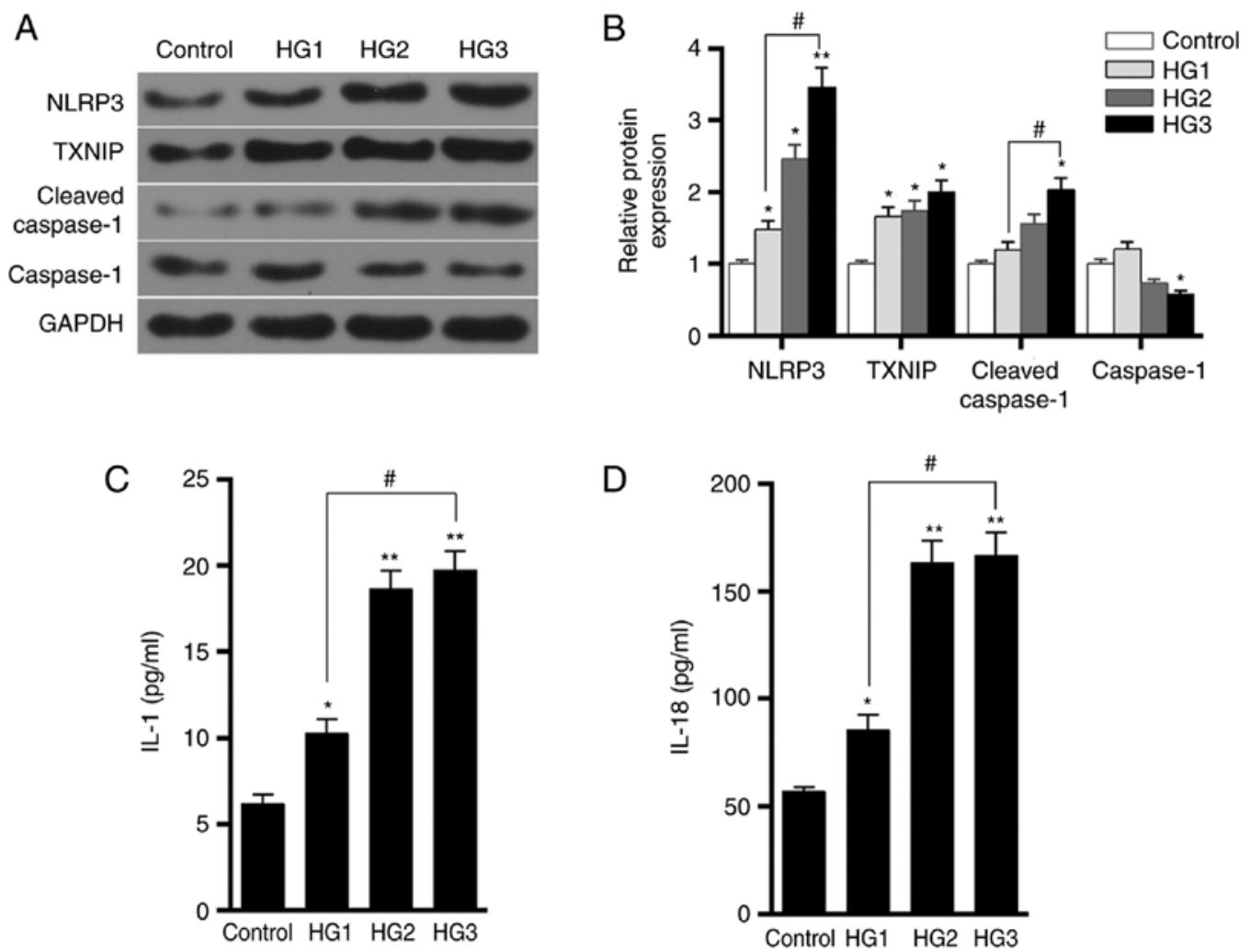

Figure 4. Effects of different concentrations of glucose on TXNIP expression and the activation of NLRP3 inflammasome in HK-2 cells. (A) Western blots and (B) quantified expression levels of TXNIP, NLRP3, cleaved-caspase-1 and caspase-1 proteins in the control, HG1, HG2 and HG3 groups, which were treated with 5.5, 15, 30 and $50 \mathrm{mmol} / 1$ glucose, respectively. (C) IL-1 and (D) IL-18 expression levels in the four groups were tested using ELISA. "P<0.05 and ${ }^{* *} \mathrm{P}<0.01$, vs. control group; ${ }^{*} \mathrm{P}<0.05$. TXNIP, thioredoxin interacting protein; NLRP3, NOD-like receptor protein 3; IL, interleukin.

were significantly lower in the siTXNIP + HG group in comparison with those in the $\mathrm{NC}+\mathrm{HG}$ groups $(\mathrm{P}<0.05)$. No significant differences were detected in caspase-1 protein expression among the different groups (Fig. 6A and B). 
A
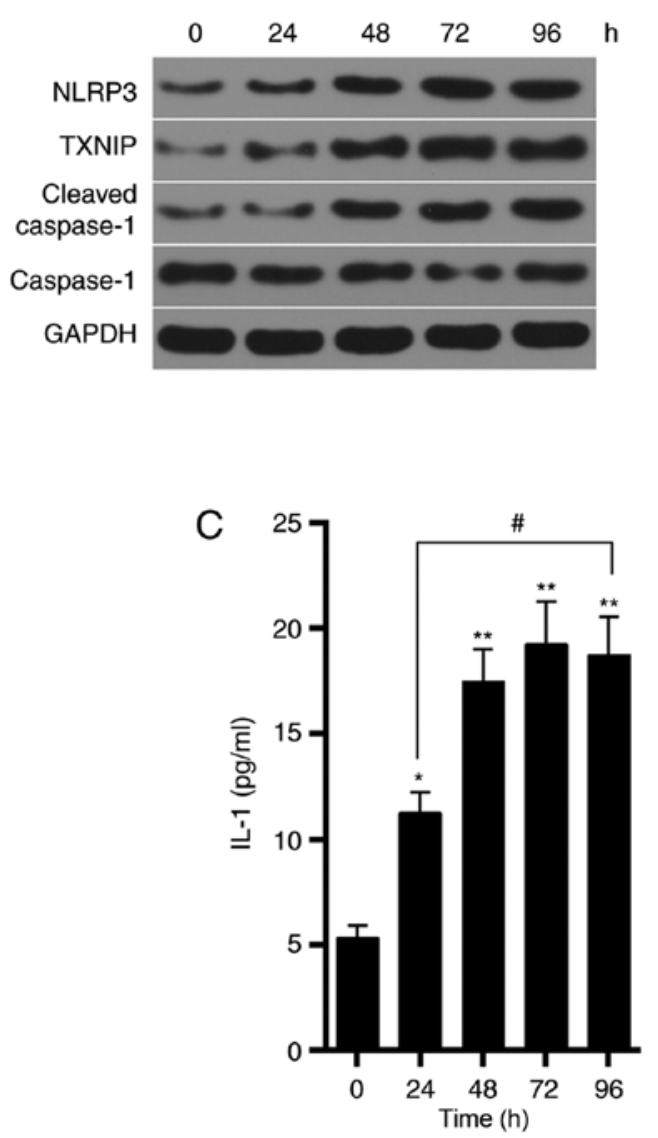

B

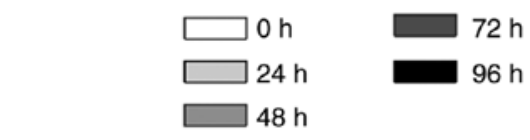

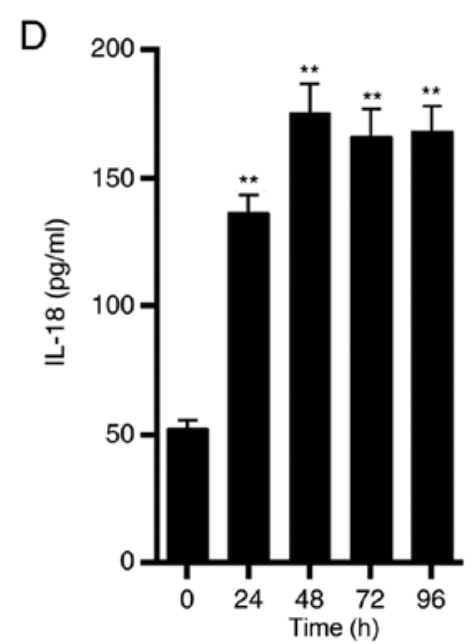

Figure 5. Effects of glucose on TXNIP expression and activation of NLRP3 inflammasome in HK-2 cells at different times. Cells were incubated with $50 \mathrm{mmol} / 1$ glucose for $0,24,48,72$ and $96 \mathrm{~h}$. (A) Western blots and (B) quantified expression levels of TXNIP, NLRP3, cleaved-caspase-1 and caspase-1 proteins at different time points. (C) IL-1 and (D) IL-18 expression levels were detected by ELISA. ${ }^{*} \mathrm{P}<0.05$ and ${ }^{* *} \mathrm{P}<0.01$, vs. control ( 0 h) group; ${ }^{\#} \mathrm{P}<0.05$ and ${ }^{\# \#} \mathrm{P}<0.01$. TXNIP, thioredoxin interacting protein; NLRP3, NOD-like receptor protein 3; IL, interleukin.

Furthermore, the concentrations of IL-1 and IL-18 in each group were detected using ELISA. IL-1 and IL-18 expression levels in $\mathrm{HG}$ and $\mathrm{NC}+\mathrm{HG}$ groups were higher compared with those in control group $(\mathrm{P}<0.01)$. However, IL-1 and IL-18 in the siTXNIP + HG group were significantly lower than those in the $\mathrm{NC}+\mathrm{HG}$ groups $(\mathrm{P}<0.05$; Fig. $6 \mathrm{C}$ and $\mathrm{D})$. These observations suggested that silencing TXNIP gene inhibited the expression levels of IL-1 and IL-18.

Effects of TXNIP gene silencing on oxidation of HK-2 cells under high-glucose conditions. The expression levels of catalase and MnSOD protein and mRNA in the control, $\mathrm{HG}, \mathrm{NC}+\mathrm{HG}$, siTXNIP and siTXNIP + HG groups were detected by western blot analysis and RT-qPCR, respectively. The results revealed that the protein and mRNA expression levels of catalase and MnSOD significantly decreased in the $\mathrm{HG}$ and $\mathrm{NC}+\mathrm{HG}$ groups compared with the control $(\mathrm{P}<0.01)$. Subsequent to silencing of the TXNIP gene in cells cultured under high glucose, the protein and mRNA expression levels of catalase and MnSOD were significantly higher in comparison with those in the $\mathrm{NC}+\mathrm{HG}$ group $(\mathrm{P}<0.05$; Fig. 7A-C). This indicated that the high-glucose environment inhibited the expression levels of antioxidant genes, while silencing of the TXNIP gene promoted the expression of antioxidant genes.
Flow cytometry was subsequently applied to detect the expression level of ROS in each group. The results revealed that the ROS level increased in the $\mathrm{HG}$ and $\mathrm{NC}+\mathrm{HG}$ groups, when compared with the control group $(\mathrm{P}<0.01)$. However, the ROS level in the siTXNIP + HG group was significantly lower in comparison with that in the $\mathrm{NC}+\mathrm{HG}$ group $(\mathrm{P}<0.05$; Fig. 7D and E). These results suggested that the high-glucose environment promoted the production of ROS, while TXNIP gene silencing was able to markedly reduce the ROS levels induced by high glucose.

\section{Discussion}

Kidney injury is one of the most common complications of diabetes (14). Currently, the mechanism of DN is not yet completely clear, and research has focused on the pathogenesis and treatment of DN in recent years. Streptozotocin is known to degenerate islet $\beta$-cells and causes diabetes (15), and streptozotocin-induced diabetic animal models are widely used in studies conducted on human diabetes (16). The results of the present study revealed that, following streptozotocin injection, the blood glucose levels of rats were $>250 \mathrm{mg} / \mathrm{dl}$, while H\&E staining also demonstrated kidney damage in the DN group. This suggested that the streptozotocin-induced 
A
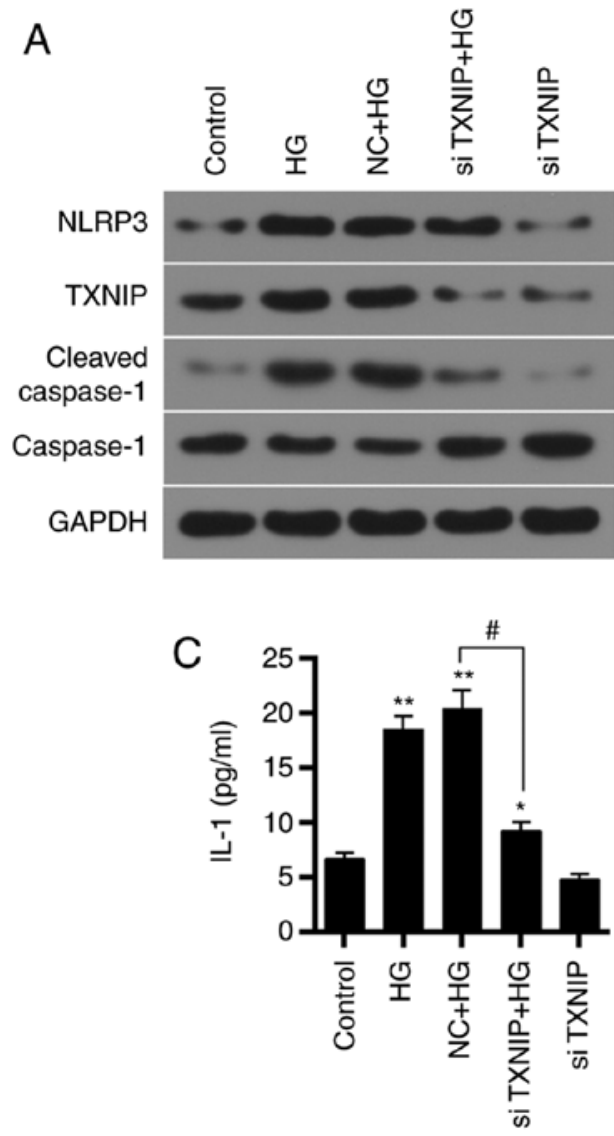

B
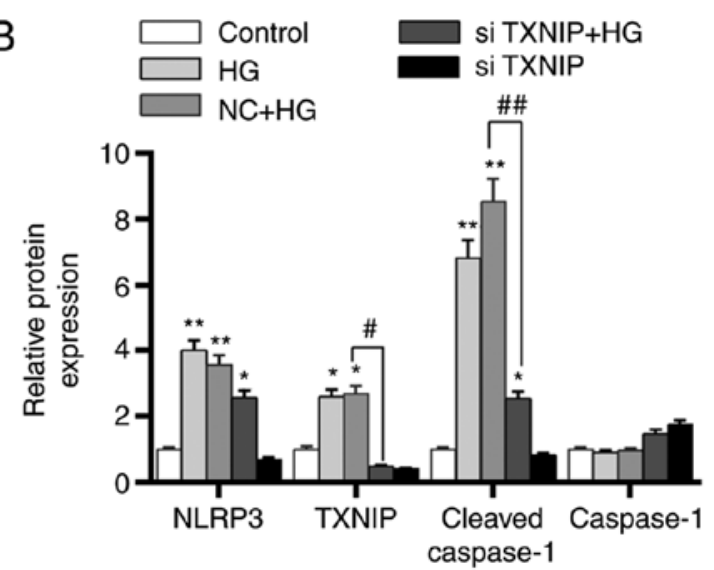

$\mathrm{D}$

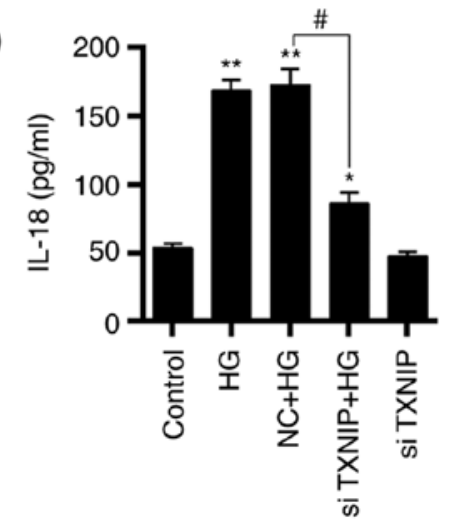

Figure 6. Effects of TXNIP gene silencing on NLRP3 inflammasome activation-associated proteins and inflammation-associated factors. (A) Western blots and (B) quantified expression levels of TXNIP, NLRP3, cleaved-caspase-1 and caspase-1 proteins in the control, HG, NC + HG, siTXNIP and siTXNIP + HG groups. (C) IL-1 and (D) IL-18 expression levels in each group were detected by ELISA. ${ }^{*} \mathrm{P}<0.05$ and ${ }^{* *} \mathrm{P}<0.01$, vs. control group; ${ }^{\#} \mathrm{P}<0.05$ and ${ }^{\# \# ~} \mathrm{P}<0.01$. TXNIP, thioredoxin interacting protein; NLRP3, NOD-like receptor protein 3; IL, interleukin; HG, high glucose; NC, negative control siRNA; si-, small interfering RNA.

diabetic kidney injury model was successfully constructed and could be used for further experiments.

Inflammation is an important factor in the development of diabetes (17), and extensive inflammatory lesions are the pathological basis of DN $(18,19)$. Studies have reported that overexpression of IL-18 increased neutrophil infiltration and worsened kidney damage $(20,21)$. NLRP3 inflammasome can activate caspase- 1 through ASC, and caspase- 1 then further cleaves and activates IL-1 and IL-18 $(22,23)$. At present, it is known that NLRP3 inflammasome activation participates in the process of DN $(4,24)$. However, the mechanism underlying the activation of NLRP3 inflammasome in DN remains unclear. ROS generation is considered as a necessary condition for the activation of NLRP3 inflammasome (25). TXNIP can regulate renal tubular and mitochondrial autophagy in DN via the mammalian target of rapamycin signaling pathway and increase oxidative stress levels (26).

Therefore, the present study conducted SP staining and western blot analysis to detect the expression characteristics of TXNIP and NLRP3 in DN rats. The results demonstrated that TXNIP and NLRP3 were overexpressed in the kidneys of DN rats, while the levels of IL-1 and IL-18, which are downstream of NLRP3 inflammasome, were also significantly increased. This is consistent with previous research results (27), indicating that the occurrence and development of DN is associated with the overexpression of TXNIP and activation of NLRP3 inflammasome. To further explore the action mechanism of TXNIP on the activation of NLRP3 inflammasomes, the levels of antioxidant genes, SOD and MDA in the kidney of DN rats were examined. The results revealed that catalase and MnSOD protein and mRNA expression levels were significantly reduced. In addition, SOD expression levels were downregulated, whereas MDA levels were upregulated. Previous studies have demonstrated that NLRP3 is able to directly act on kidney tubular epithelial cells involved in kidney injury during renal ischemia-reperfusion (28), and no evident protective effect was observed on the kidney when mouse NLRP3 gene was knocked out (29). Another study reported that elevated blood glucose caused increased levels of oxidative stress in the body (30). These observations suggested that the activation of NLRP3 inflammasomes was associated with the level of oxidative stress.

To investigate the possible activation mechanism of the NLRP3 inflammasome, the HK-2 cell line was used to mimic the high-glucose environment in vitro and explore the effect of the high-glucose concentration on HK-2 cells. The results indicated that the high-glucose environment was able to upregulate the expression of TXNIP and the activation of NLRP3 inflammasomes in a dose-dependent and time-dependent manner, respectively, while it also promoted the secretion of IL-1 and IL-18 in HK-2 cells. A previous study has reported that a high-glucose concentration and lipopolysaccharides 

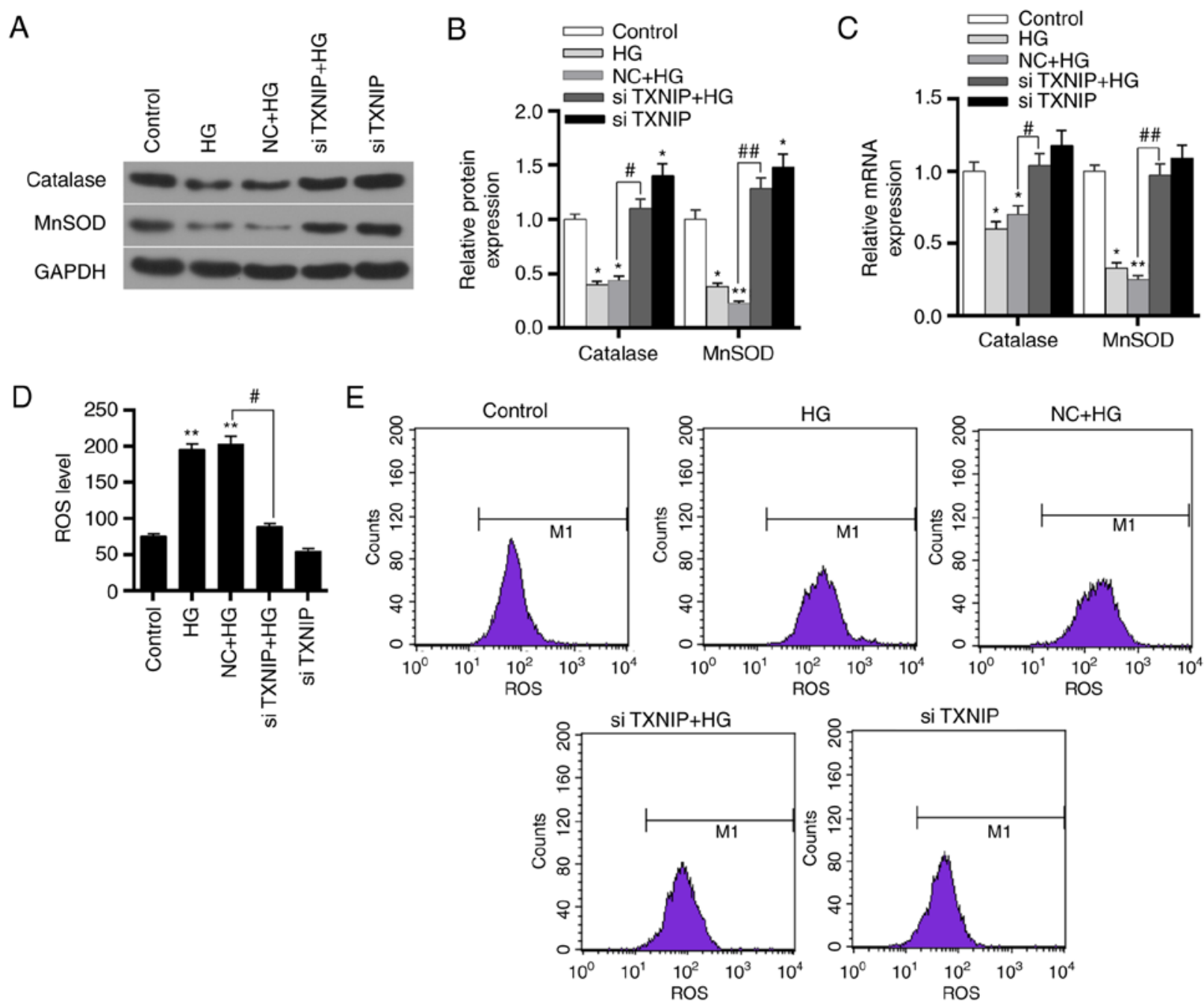

Figure 7. Effects of TXNIP gene silencing on antioxidant gene expression and ROS levels in HK-2 cells. (A) Western blots and (B) quantified protein expression levels of catalase and MnSOD in the control, HG, NC + HG, siTXNIP + HG and siTXNIP groups, detected using western blot analysis. (C) mRNA expression levels of catalase and MnSOD in the different groups, detected using reverse transcription-quantitative polymerase chain reaction. (D) ROS levels in each group and (E) flow cytometry applied to detect these levels. ${ }^{\mathrm{P}}<0.05$ and ${ }^{* *} \mathrm{P}<0.01$, vs. control group; ${ }^{\#} \mathrm{P}<0.05$ and ${ }^{\# \#} \mathrm{P}<0.01$. TXNIP, thioredoxin interacting protein; ROS, reactive oxygen species; HG, high glucose; NC, negative control siRNA; si-, small interfering RNA.

significantly induced the mRNA and protein levels of TXNIP, NLRP3, procaspase-1 and IL-1 $\beta$ in mesangial cells (31). Another study has also identified that the high-glucose conditions also caused human retinal microvascular endothelial cell damage via the TXNIP/NLRP3 pathway (32). This suggested that the high-glucose environment may act directly on human kidney tubular epithelial cells and activate NLRP3 inflammasomes, leading to inflammatory damage, which may be associated with their ability to promote TXNIP expression levels.

To further study the role of TXNIP gene in DN and the mechanism of regulating NLRP3 inflammasome, RNA interference technology was applied to silence TXNIP gene, and the effect of TXNIP gene silencing on NLRP3 inflammasome in the high-glucose environment was investigated. The results revealed that TXNIP gene expression was upregulated in the high-glucose environment and that NLRP3 inflammasome was overactivated. Interference with siTXNIP plasmids inhibited the effect of high-glucose on TXNIP, as well as reduced the activation of NLRP3 inflammasome and the secretion of downstream IL-1 and IL-18. This suggests that TXNIP silencing inhibited NLRP3 inflammasome activation-associated proteins. However, the action mechanism of TXNIP in DN remained unclear.

To further investigate the mechanism through which TXNIP activates the NLRP3 inflammasome, the effects of TXNIP gene silencing on the antioxidant genes and ROS were investigated in HK-2 cells under a the high-glucose condition. The results demonstrated that the high-glucose environment inhibited the expression of antioxidant genes catalase and MnSOD in HK-2 cells, and increased ROS levels. Following the silencing of TXNIP expression, catalase and MnSOD protein and mRNA levels were significantly increased and ROS production was inhibited. It has previously been suggested that TXNIP is the most important endogenous inhibitor of thioredoxin and constitutes an important part of the oxygen reduction system in the body (33). A recent study has identified that ROS was able to activate the NLRP3/IL-1 $\beta$ pathway by upregulating the level of TXNIP, therefore aggravating the inflammatory response (34). Zhou et al (35) observed that ROS induced TXNIP to directly bind to NLRP3 and activated NLRP3 inflammasome; however, related research has mainly focused on metabolic diseases and glomerular diseases. A previous study has reported that TXNIP 
upregulated oxidative stress by inhibiting TRX, which leads to an increase at ROS levels (36). Another study suggested that, under high-glucose conditions, downregulation of TXNIP levels inhibited ROS production via the p38 mitogen activated protein kinase and extracellular signal-regulated kinase pathways (36). The results of Li et al (37) demonstrated that overexpression of TXNIP was able to inhibit liver cancer cell proliferation and induce apoptosis by triggering mitochondria-mediated ROS production. Combined with the results of the present study, it is suggested that ROS may interact with TXNIP in DN. In diabetic rats, high-glucose levels caused TXNIP gene overexpression and increased ROS levels by inhibiting the expression of antioxidant genes. Under the action of ROS, TXNIP further activated the NLRP3 inflammasome and caused kidney damage. TXINP silencing upregulated the expression of antioxidant genes, while it reduced not only the level of oxidative stress in the body and the expression of NLRP3, but also the activation of NLRP3 inflammasomes and the damage of the inflammatory response to the kidney (38-40).

In conclusion, the findings of the present study suggest that a high-glucose environment may promote the expression of TXNIP, increasing the body's oxidative stress level and inducing DN by activating the NLRP3 inflammasome. TXNIP gene silencing promoted the expression of antioxidant factors and decreased ROS levels, reducing the inflammatory levels by inhibiting NLRP3 inflammasome activation. This suggested that the occurrence and development of DN was closely associated with the activation of NLRP3 inflammasome caused by TXNIP, and thus TXNIP may provide a new approach for the treatment of DN.

\section{Acknowledgements}

Not applicable.

\section{Funding}

No funding was received.

\section{Availability of data and materials}

The analyzed data sets generated during the study are available from the corresponding author on reasonable request.

\section{Authors' contributions}

CG and HW made substantial contributions to the study conception and design. HW and HD were responsible for data acquisition, data analysis and interpretation. HD drafted and critically revised the manuscript for important intellectual content. SL and HW agreed to be accountable for all aspects of the work in ensuring that questions related to the accuracy or integrity of the work are appropriately investigated and resolved. All authors approved the final version of the manuscript.

\section{Ethics approval and consent to participate}

All protocols were approved by the Institutional Animal Care and Use Committee of the First Hospital of Jilin University, and the China Council on Animal Care.

\section{Patient consent for publication}

Not applicable.

\section{Competing interests}

The authors declare that they have no conflicts of interest.

\section{References}

1. Guariguata L, Whiting DR, Hambleton I, Beagley J, Linnenkamp U and Shaw JE: Global estimates of diabetes prevalence for 2013 and projections for 2035. Diabetes Res Clin Pract 103: 137-149, 2014.

2. Duran-Salgado MB and Rubio-Guerra AF: Diabetic nephropathy and inflammation. World J Diabetes 5: 393-398, 2014.

3. Beisswenger PJ, Drummond KS, Nelson RG, Howell SK, Szwergold BS and Mauer M: Susceptibility to diabetic nephropathy is related to dicarbonyl and oxidative stress. Diabetes 54: 3274-3281, 2005.

4. Shahzad K, Bock F, Dong W, Wang H, Kopf S, Kohli S, Al-Dabet MM, Ranjan S, Wolter J, Wacker C, et al: Nlrp3-inflammasome activation in non-myeloid-derived cells aggravates diabetic nephropathy. Kidney Int 87: 74-84, 2015.

5. Sun B, Wang X, Ji Z, Li R and Xia T: NLRP3 inflammasome activation induced by engineered nanomaterials. Small 9: 1595-1607, 2013

6. Abderrazak A, Syrovets T, Couchie D, El Hadri K, Friguet B, Simmet T and Rouis M: NLRP3 inflammasome: From a danger signal sensor to a regulatory node of oxidative stress and inflammatory diseases. Redox Biol 4: 296-307, 2015.

7. Granata S, Masola V, Zoratti E, Scupoli MT, Baruzzi A, Messa M, Sallustio F, Gesualdo L, Lupo A and Zaza G: NLRP3 inflammasome activation in dialyzed chronic kidney disease patients. PLoS One 10: e0122272, 2015.

8. Hutton HL, Ooi JD, Holdsworth SR and Kitching AR: The NLRP3 inflammasome in kidney disease and autoimmunity. Nephrology (Carlton) 21: 736-744, 2016.

9. Ogata FT, Batista WL, Sartori A, Gesteira TF, Masutani H, Arai RJ, Yodoi J, Stern A and Monteiro HP: Nitrosative/oxidative stress conditions regulate thioredoxin-interacting protein (TXNIP) expression and thioredoxin-1 (TRX-1) nuclear localization. PLoS One 8: e84588, 2013.

10. Mohamed IN, Hafez SS, Fairaq A, Ergul A, Imig JD and El-Remessy AB: Thioredoxin-interacting protein is required for endothelial NLRP3 inflammasome activation and cell death in a rat model of high-fat diet. Diabetologia 57: 413-423, 2014.

11. Sonne SB, Perrett RM, Nielsen JE, Baxter MA, Kristensen DM, Leffers H, Hanley NA and Rajpert-De-Meyts E: Analysis of SOX2 expression in developing human testis and germ cell neoplasia. Int J Dev Biol 54: 755-760, 2010.

12. Lim J, Goriely A, Turner GD, Ewen KA, Jacobsen GK, Graem N, Wilkie AO and Rajpert-De Meyts E: OCT2, SSX and SAGE1 reveal the phenotypic heterogeneity of spermatocytic seminoma reflecting distinct subpopulations of spermatogonia. J Pathol 224: 473-483, 2011.

13. Livak KJ and Schmittgen TD: Analysis of relative gene expression data using real-time quantitative PCR and the 2(-Delta Delta C(T)) method. Methods 25: 402-408, 2001.

14. Heneghan HM, Cetin D, Navaneethan SD, Orzech N, Brethauer SA and Schauer PR: Effects of bariatric surgery on diabetic nephropathy after 5 years of follow-up. Surg Obes Relat Dis 9: 7-14, 2013.

15. Lenzen S: The mechanisms of alloxan-and streptozotocin-induced diabetes. Diabetologia 51: 216-226, 2008

16. Elias D, Prigozin H, Polak N, Rapoport M, Lohse AW and Cohen IR: Autoimmune diabetes induced by the beta-cell toxin STZ. Immunity to the $60-\mathrm{kDa}$ heat shock protein and to insulin. Diabetes 43: 992-998, 1994.

17. Hanley AJ, Festa A, D'Agostino RB Jr, Wagenknecht LE, Savage PJ, Tracy RP, Saad MF and Haffner SM: Metabolic and inflammation variable clusters and prediction of type 2 diabetes: Factor analysis using directly measured insulin sensitivity. Diabetes 53: 1773-1781, 2004.

18. Wada $\mathbf{J}$ and Makino $\mathrm{H}$ : Inflammation and the pathogenesis of diabetic nephropathy. Clin Sci (Lond) 124: 139-152, 2013. 
19. Elsherbiny NM, Al-Gayyar MM and Abd El Galil KH: Nephroprotective role of dipyridamole in diabetic nephropathy: Effect on inflammation and apoptosis. Life Sci 143: 8-17, 2015.

20. Gonul Y, Kazandi S, Kocak A, Ahsen A, Bal A, Karavelioglu A, Hazman O, Turamanlar O, Kokulu S and Yuksel S: Interleukin-18 binding protein pretreatment attenuates kidney injury induced by hepatic ischemia reperfusion. Am J Med Sci 352: 200-207, 2016.

21. Zhen J, Zhang L, Pan J, Ma S, Yu X, Li X, Chen S and Du W: AIM2 mediates inflammation-associated renal damage in hepatitis B virus-associated glomerulonephritis by regulating caspase-1, IL-1beta, and IL-18. Mediators Inflamm 2014: 190860, 2014.

22. Lin YC, Huang DY, Wang JS, Lin YL, Hsieh SL, Huang KC and Lin W: Syk is involved in NLRP3 inflammasome-mediated caspase-1 activation through adaptor ASC phosphorylation and enhanced oligomerization. J Leukoc Biol 97: 825-835, 2015.

23. Karmakar M, Katsnelson M, Malak HA, Greene NG, Howell SJ, Hise AG, Camilli A, Kadioglu A, Dubyak GR and Pearlman E: Neutrophil IL-1 $\beta$ processing induced by pneumolysin is mediated by the NLRP3/ASC inflammasome and caspase-1 activation and is dependent on K+ efflux. J Immunol 194: 1763-1775, 2015.

24. Chen K, Zhang J, Zhang W, Zhang J, Yang J, Li K and He Y: ATP-P2X4 signaling mediates NLRP3 inflammasome activation: A novel pathway of diabetic nephropathy. Int J Biochem Cell Biol 45: 932-943, 2013.

25. Bauernfeind F, Bartok E, Rieger A, Franchi L, Nunez G and Hornung V: Cutting edge: Reactive oxygen species inhibitors block priming, but not activation, of the NLRP3 inflammasome. J Immunol 187: 613-617, 2011.

26. Huang C, Zhang Y, Kelly DJ, Tan CY, Gill A, Cheng D, Braet F, Park JS, Sue CM, Pollock CA and Chen XM: Thioredoxin interacting protein (TXNIP) regulates tubular autophagy and mitophagy in diabetic nephropathy through the mTOR signaling pathway. Sci Rep 6: 29196, 2016.

27. Samra YA, Said HS, Elsherbiny NM, Liou GI, El-Shishtawy MM and Eissa LA: Cepharanthine and piperine ameliorate diabetic nephropathy in rats: Role of NF-kappaB and NLRP3 inflammasome. Life Sci 157: 187-199, 2016.

28. Shigeoka AA, Mueller JL, Kambo A, Mathison JC, King AJ, Hall WF, Correia Jda S, Ulevitch RJ, Hoffman HM and McKay DB: An inflammasome-independent role for epithelial-expressed Nlrp3 in renal ischemia-reperfusion injury. J Immunol 185: 6277-6285, 2010.

29. Kim HJ, Lee DW, Ravichandran K, O Keys D, Akcay A, Nguyen Q, He Z, Jani A, Ljubanovic D and Edelstein CL: NLRP3 inflammasome knockout mice are protected against ischemic but not cisplatin-induced acute kidney injury. J Pharmacol Exp Ther 346: 465-472, 2013

30. Zhang W, Zhao S, Li Y, Peng G and Han P: Acute blood glucose fluctuation induces myocardial apoptosis through oxidative stress and nuclear factor-kB activation. Cardiology 124: 11-17, 2013.
31. Feng H, Gu J, Gou F, Huang W, Gao C, Chen G, Long Y, Zhou X, Yang M, Liu S, et al: High glucose and lipopolysaccharide prime NLRP3 inflammasome via ROS/TXNIP pathway in mesangial cells. J Diabetes Res 2016: 6973175, 2016.

32. Chen W, Zhao M, Zhao S, Lu Q, Ni L, Zou C, Lu L, Xu X, Guan H, Zheng Z and Qiu Q: Activation of the TXNIP/NLRP3 inflammasome pathway contributes to inflammation in diabetic retinopathy: A novel inhibitory effect of minocycline. Inflamm Res 66: 157-166, 2017.

33. Devi TS, Hosoya K, Terasaki T and Singh LP: Critical role of TXNIP in oxidative stress, DNA damage and retinal pericyte apoptosis under high glucose: Implications for diabetic retinopathy. Exp Cell Res 319: 1001-1012, 2013.

34. Han Y, Xu X, Tang C, Gao P, Chen X, Xiong X, Yang M, Yang S, Zhu X, Yuan S, et al: Reactive oxygen species promote tubular injury in diabetic nephropathy: The role of the mitochondrial ros-txnip-nlrp3 biological axis. Redox Biol 16: 32-46, 2018.

35. Zhou R, Tardivel A, Thorens B, Choi I and Tschopp J: Thioredoxin-interacting protein links oxidative stress to inflammasome activation. Nat Immunol 11: 136-140, 2010.

36. Li W, Wu Z, Ma Q, Liu J, Xu Q, Han L, Duan W, Lv Y, Wang F, Reindl KM and Wu E: Hyperglycemia regulates TXNIP/TRX/ROS axis via p38 MAPK and ERK pathways in pancreatic cancer. Curr Cancer Drug Targets 14: 348-356, 2014.

37. Li J, Yue Z, Xiong W, Sun P, You K and Wang J: TXNIP overexpression suppresses proliferation and induces apoptosis in SMMC7221 cells through ROS generation and MAPK pathway activation. Oncol Rep 37: 3369-3376, 2017.

38. Chang A, Ko K and Clark MR: The emerging role of the inflammasome in kidney diseases. Curr Opin Nephrol Hypertens 23 204-210, 2014

39. Shirasuna K, Takano H, Seno K, Ohtsu A, Karasawa T, Takahashi M, Ohkuchi A, Suzuki H, Matsubara S, Iwata H and Kuwayama T: Palmitic acid induces interleukin-lbeta secretion via NLRP3 inflammasomes and inflammatory responses through ROS production in human placental cells. J Reprod Immunol 116: 104-112, 2016.

40. Xia M, Abais JM, Koka S, Meng N, Gehr TW, Boini KM and Li PL: Characterization and activation of NLRP3 inflammasomes in the renal medulla in mice. Kidney Blood Press Res 41: 208-221, 2016.

This work is licensed under a Creative Commons Attribution-NonCommercial-NoDerivatives 4.0 International (CC BY-NC-ND 4.0) License. 